Research Paper

\title{
Carum copticum and Thymus vulgaris oils inhibit virulence in Trichophyton rubrum and Aspergillus spp
}

\author{
Mohd Sajjad Ahmad Khan ${ }^{1,2}$, Iqbal Ahmad ${ }^{1}$, Swaranjit Singh Cameotra ${ }^{2}$ \\ ${ }^{1}$ Department of Agricultural Microbiology, Aligarh Muslim University, Aligarh, India. \\ ${ }^{2}$ Institute of Microbial Technology, Chandigarh, India.
}

Submitted: August 17, 2012; Approved: September 9, 2013.

\begin{abstract}
Emergence of drug-resistant strains has demanded for alternative means of combating fungal infections. Oils of Carum copticum and Thymus vulgaris have long been used in ethnomedicine for ailments of various fungal infections. Since their activity has not been reported in particular against drug-resistant fungi, this study was aimed to evaluate the effects of oils of C. copticum and T. vulgaris on the growth and virulence of drug-resistant strains of Aspergillus spp. and Trichophyton rubrum. The gas chromatography-mass spectrometry analysis revealed thymol constituting $44.71 \%$ and $22.82 \%$ of $T$. vulgaris and C. copticum, respectively. Inhibition of mycelial growth by essential oils was recorded in the order of thymol $>T$. vulgaris $>C$. copticum against the tested strains. RBC lysis assay showed no tested oils to be toxic even up to concentration two folds higher than their respective MFCs. Thymol exhibited highest synergy in combination with fluconazole against Aspergillus fumigatus MTCC2550 (FICI value 0.187) and T. rubrum IOA9 (0.156) as determined by checkerboard method. Thymol and $T$. vulgaris essential oil were equally effective against both the macro and arthroconidia growth (MIC $72 \mu \mathrm{g} / \mathrm{mL}$ ). A $>80 \%$ reduction in elastase activity was recorded for $A$. fumigatus MTCC2550 by C. copticum, T. vulgaris oils and thymol. The effectiveness of these oils against arthroconidia and synergistic interaction of thymol and $T$. vulgaris with fluconazole can be exploited to potentiate the antifungal effects of fluconazole against drug-resistant strains of $T$. rubrum and Aspergillus spp.
\end{abstract}

Key words: anti-elastase activity, arthroconidia, synergy, thymol, virulence.

\section{Introduction}

Fungal infections caused by various pathogenic and opportunistic strains are on the rise in the different parts of the world. This is primarily due to growing number of high risk patients particularly immunocompromised. Filamentous fungi including Aspergillus fumigatus and dermatophytes are common reported pathogens (Vermount et al., 2008; Dagenais and Keller, 2009). Invasive aspergillosis caused by Aspergillus spp. in immunocompromised hosts may cause morbidity and mortality in a range from 40 to $90 \%$ in high risk populations (Dagenais and Keller, 2009). Whereas, increased incidence of dermatophytoses, infections of hair, skin and nails, caused by Trichophyton spp., have been reported in recent years especially in the tropical countries (Vazquez, 2003). Such infections are not life-threatening; however, both immunocompetent and immunosuppressed persons are affected. Such infections have increased considerably among pediatric and geriatric populations (Monod, 2008) and can become serious in immunocompromised patients resulting in invasive infections (Sokovic et al., 2008). Hence, the management of these fungal infections would be a definite challenge to mankind.

In spite of introduction of newer antifungal drugs, such fungal infections have been threatened by the development of drug resistant strains, host toxicity and variable drug bioavailability (Barker and Rogers, 2006). Also, these drugs are costly and not affordable to a larger section of human population across the globe (Barker and Rogers, 2006; Baddley and Pappas, 2007; Ahmad et al., 2010). Therefore, 
alternative strategies have to be explored and exploited. One of the approaches is to search for novel broad spectrum antifungal compounds from natural products including medicinal plants which can be used alone or in combiantion with known antifungal drugs. Combination therapy has potential advantages over monotherapy in terms of reducing dose related toxicity and emergence of drug resistance (Baddley and Pappas, 2007). However, in addition to killing fungi, attenuation of virulence factors has been considered as novel antifungal drug action. Targeting virulence attributes is an attracting attention to develop antifungal agent based on anti-pathogenic drug principle (Gauwerky et al., 2009). Establishment of infection by fungi depends on the host cell interaction with complex interplay of secretion of virulence factors mainly proteinases including elastinases, keratinases, gelatinases and lipases such as esterases and phospholipases. These extracellular enzymes have been identified as putative virulence factors in Aspergillus spp. and Trichophyton spp. by assisting these organisms to degrade structural barrier and immune cells, and to obtain nutrient and in establishing infections (Voltan et al., 2008). In addition, arthroconidia are considered as the primary cause of infection and pathogenesis by dermatophytes and often produced under in vivo condition (Yazdanparast and Barton, 2006). Arthroconidia are dormant fungal conidia that are metabolically active cells enriched with lipid-containing vacuoles and intracellular organelles and are highly resistant to some antifungal agents and adverse environmental conditions (Yazdanparast and Barton, 2006; Barros et al., 2007). This variation in drug sensitivity due to vegetative or arthroconidial form in T. rubrum is the main reason for failure of dermatophytosis treatment in clinical practice.

Plant products traditionally being used in ethnomedicine have been expected to deliver newer antifungal compounds. In particular, oils of $C$. copticum and T. vulgaris have been well documented for ethnomedicinal values in traditional medicine (Pina-Vaz et al., 2004; Bairwa et al., 2012). Antifungal activities of these essential oils against Aspergillus spp. and Trichophyton spp. have been reported by several workers (Zacchino et al., 1999; Cavaleiro et al., 2006; Tullio et al., 2007; Bajpai et al., 2009; Khan and Ahmad, 2011). But interaction of these oils with antifungal drugs has not been explored. Moreover, the influence of these oils on fungal virulence factors synthesis and activity are also not yet explored or poorly known.

In lieu of this, the present study was aimed to determine in vitro growth inhibition of $T$. rubrum and Aspergillus spp. by the oils of C. copticum and T. vulgaris and their major component thymol. These agents were also assessed for their synergistic interaction with antifungal drug fluconazole. Further, to explore anti-pathogenic drug principle of these oils, inhibition of elastase and keratinase enzymes was examined.

\section{Materials and Methods}

\section{Plant essential oils and drugs}

Essential oils (C. copticum; ajowan and T. vulgaris; thyme) were purchased from Aroma Sales Corporation, New Delhi. Thymol (98\% purity) was obtained from Himedia Ltd., Mumbai. The drug powder of fluconazole was purchased from Pfizer, Mumbai, India. Stock solution of fluconazole was prepared in dimethyl sulphoxide (DMSO) at a concentration of $25 \mathrm{mg} / \mathrm{mL}$ and stored at $-20{ }^{\circ} \mathrm{C}$ until used. The purity of oils and active compounds was determined by physico-chemical analyses such as specific gravity, refractive index, optical rotation and solubility in alcohol at Fragrance and Flavour Development Centre, Kannauj, India. The suspensions of essential oils were prepared by diluting ten times in $1 \%$ DMSO and used in assays at concentrations ranging from 4.5 to $2304 \mu \mathrm{g} / \mathrm{mL}$.

\section{Fungal strains}

Aspergillus fumigatus MTCC2550 was purchased from Microbial Type Culture Collection, India; A. niger IOA3 and T. rubrum IOA9 were collected from Jawaharlal Nehru Medical College and Hospital, AMU, Aligarh, India and are maintained at the departmental culture collection. These fungal strains were resistant to azoles including fluconazole and itraconazole (Khan and Ahmad, 2011).

\section{Gas chromatography and gas chromatography-mass spectrometry analysis}

The percentage composition of oils of C. copticum and $T$. vulgaris was determined by GC-FID and the compounds were identified by GC-MS. GC analysis was carried out on a Shimadzu 2010 Gas Chromatograph equipped with an FID and $25 \mathrm{~m} \times 0.25 \mathrm{~mm} \times 0.25 \mu \mathrm{m}$ WCOT column coated with diethylene glycol (AB-Innowax, 7031428, Japan). Injector temperature was set at $270{ }^{\circ} \mathrm{C}$ and detector at $280^{\circ} \mathrm{C}$. Nitrogen was used as a carrier gas at a flow rate of $3.0 \mathrm{~mL} / \mathrm{min}$ at a column pressure of $74.9 \mathrm{kPa} .0 .2 \mu \mathrm{L}$ of sample were injected into column with a split ratio of 90.0 . The linear temperature program of $60{ }^{\circ} \mathrm{C}$ to $230{ }^{\circ} \mathrm{C}$ set at a rate of $3{ }^{\circ} \mathrm{C} / \mathrm{min}$ with hold time at $230^{\circ} \mathrm{C}$ for $10 \mathrm{~min}$. The samples were than analyzed on the same Shimadzu instrument fitted with the same column and following the same temperature program as above. MS parameters used were: ionisation voltage (EI) $70 \mathrm{eV}$, peak width $2 \mathrm{~s}$, mass range 40-600 amu and detector voltage $1.5 \mathrm{~V}$. Results were based on GC-FID. Peak identification was carried out by comparison of the mass spectra with database of NIST05 and Wiley8 libraries. Identification of compounds was confirmed by comparison of their relative retention indices with literature values (Davies, 1990).

\section{Assays for determination of antifungal activity}

Antifungal effect of essential oils was determined in terms of MIC and inhibition of biomass in liquid medium and mycelial radial growth on solid medium as described below. 


\section{Determination of minimum inhibitory concentration}

The minimum inhibitory concentration of oils of $C$. copticum, T. vulgaris, thymol and fluconazole was determined as described elsewhere (Khan and Ahmad, 2011).

\section{Inhibition of mycelial biomass production}

Method of Shafique et al. (2011) with slight modification was used to assess the effect of essential oils and drugs on the production of mycelial biomass by tested fungi. Briefly, $50 \mathrm{~mL}$ Sabouraud dextrose broth (SDB) containing different concentrations of essential oils was inoculated with $500 \mu \mathrm{L}$ of freshly prepared conidial suspension $\left(\sim 1.5 \times 10^{6} \mathrm{cfu} / \mathrm{mL}\right)$. In the corresponding control an equal amount of distilled water was added. The flasks were incubated at $28 \pm 2{ }^{\circ} \mathrm{C}$ for 5 days. Thereafter, mycelial biomass from triplicate samples for each treatment was collected on pre-weighed Whatman filter paper No.1. Mycelial yield was determined after drying the mycelial mat at $80{ }^{\circ} \mathrm{C}$ for $24 \mathrm{~h}$. The experiments were performed three times and mean percent loss in mycelial dry weight was calculated over untreated control.

\section{Inhibition of mycelial radial growth}

Inhibition of mycelial radial growth by tested oils and drugs was determined by the method of Quiroga et al. (2004) with little modification. Briefly, a $5 \mathrm{~mm}$ diameter disc of inoculum of the tested fungi was cut from the periphery of an actively growing culture and placed onto the SDA Petri plates amended with oils and thymol. The SDA plates without oils and thymol and served as untreated controls. All the inoculated plates were incubated at $28 \pm 2{ }^{\circ} \mathrm{C}$ for 5 days. Three replicates for each combination of tested fungi and oils/thymol concentrations were used. The experiment was performed three times and the mean diameter of the radial growth of the fungi was recorded at the end of the incubation period and percent growth inhibition was calculated in comparison with untreated controls.

\section{RBC lysis assay}

The toxicity of essential oils or active compounds was evaluated by the red blood cell (RBC) lysis assay as adapted by Luize et al. (2005) with some modifications. The freshly obtained RBCs of sheep blood were washed with $1 \mathrm{~mL}$ of PBS (pH 7.0) and $4 \mathrm{~mL}$ was added to $5 \%$ $(\mathrm{w} / \mathrm{v})$ glucose solution to obtain $4 \%$ RBC suspension. $750 \mu \mathrm{L}$ of PBS containing the desired concentration of test agent was mixed with $750 \mu \mathrm{L}$ of RBC suspension in Eppendorf tubes and incubated at $37{ }^{\circ} \mathrm{C}$ for $2 \mathrm{~h}$. Triton $\mathrm{X}-100$ $(0.1 \%(\mathrm{v} / \mathrm{v})$ in PBS) was used as a positive control whereas $1 \%$ DMSO and PBS were used as negative controls. Tubes were centrifuged at $2000 \mathrm{rpm}$ for $10 \mathrm{~min}$ and the absorbances of supernatant were read at $540 \mathrm{~nm}$. Percent haemolysis was calculated as: $[\{(\mathrm{A}-\mathrm{B}) /(\mathrm{C}-\mathrm{B})\} \mathrm{X} 100]$. Where A and $\mathrm{B}$ are the absorbance values of supernatant from the test sample and PBS (solvent control) respectively and C is the absorbance value of supernatant from the sample after $100 \%$ lysis. Each experiment was performed three times in triplicate and the mean values were considered for calculation of percent haemolysis.

\section{Interaction of essential oils with fluconazole}

A checkerboard microtiter test was performed to evaluate the interaction of C. copticum, T. vulgaris oils and thymol with fluconazole against $A$. fumigatus MTCC2550 and T. rubrum IOA9. The series of two-fold dilutions, in eight numbers, of each agent were made in SDB to obtain four times the final concentration being achieved in the microtiter well. Furthermore, $50 \mu \mathrm{L}$ of each dilution of oils/compounds was added to the 96 well microtiter plates in the vertical direction, while $50 \mu \mathrm{L}$ of each dilution of fluconazole was added in the horizontal direction, so that various combinations of essential oils or active compounds and fluconazole could be achieved. Also, $100 \mu \mathrm{L}$ of conidial suspension were added to each well and plates were incubated at $30{ }^{\circ} \mathrm{C}$ for 2 days. The nature of interaction was defined quantitatively by means of fractional inhibitory concentrations (FIC) that were calculated as the MIC of the combination of essential oil or active compound with fluconazole divided by the MIC of essential oil or active compound or fluconazole alone. An FIC index (FICI) was obtained by adding both FICs. The combination result was interpreted as follows: $\mathrm{FICI} \leq 0.5$, synergistic; $>0.5-4.0$, no interaction; $>4.0$, antagonistic as described by Odds (Odds, 2003).

\section{MIC Determination against macroconidia and arthroconidia in T. rubrum}

Firstly, for the preparation of the macroconidia suspensions, cultures were grown on malt extract agar

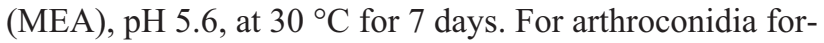
mation, a standard $\mathrm{pH} 7.5$ was employed for MEA and cultures were grown for 15 days on $5 \% \mathrm{CO} 2$ at $37^{\circ} \mathrm{C}$ in a $\mathrm{CO} 2$ incubator (Shel Lab, USA). The microscopic examinations of macroconidial and arthroconidial suspensions were conducted. Further, susceptibility of macroconidia and arthroconidia of T. rubrum IOA09 strain to C. copticum, $T$. vulgaris oils, thymol and fluconazole was tested in terms of MICs. Briefly, colonies were covered with sterile saline containing $1 \%$ Tween 80 , macroconidia or arthroconidia were harvested by sterile scraping and the solution was filtered through glass wool to remove residual hyphae or long chains of arthroconidia. The number of macroconidia or arthroconidia was estimated microscopically and the conidial suspensions were diluted in RPMI 1640 (Sigma) buffered with MOPS to obtain density of $0.5 \times 10^{5} \mathrm{cfu} / \mathrm{mL}$. The tests were performed in 96-well microtitre plates. Each microdilution well containing $100 \mu \mathrm{L}$ of the diluted $(2 \mathrm{x})$ test agent concentrations was inoculated with $100 \mu \mathrm{L}$ of the 
diluted $(2 \mathrm{x})$ macroconidial or arthroconidial suspensions. Microtitre plates were incubated at $28{ }^{\circ} \mathrm{C}$ and MICs were recorded after 5 days of incubation. MIC was defined as the lowest concentration resulting in total inhibition of visible growth. All the tests were performed three times in triplicates and mean values were considered for calculation of MICs.

\section{Assays for determination of anti-virulence activity}

\section{Detection and inhibition of elastase activity}

The method of Kothary et al. (1984) was adapted to detect the production of elastase among tested isolates; the elastase activity was determined by colorimetric assay of Sachar et al. (1955) with some modifications employing elastin congo red (Sigma) as a substrate. Briefly, $250 \mu \mathrm{L}$ each of $50 \mathrm{mM}$ sodium borate buffer $(\mathrm{pH} 8.5)$ and elastin congo red $(20 \mathrm{mg} / \mathrm{mL}$ in $50 \mathrm{mM}$ sodium borate buffer, $\mathrm{pH}$ 8.5) was mixed and vortexed at room temperature for $5 \mathrm{~min}$. Next, $250 \mu \mathrm{L}$ of each fungus was added and incubated at $37^{\circ} \mathrm{C}$ for $3 \mathrm{~h}$ at $180 \mathrm{rpm}$. Test sample was replaced by $250 \mu \mathrm{L}$ of buffer as control. After incubation, $750 \mu \mathrm{L}$ of $10 \%$ trichloro acetic acid was added to stop the reaction and kept for $30 \mathrm{~min}$ on ice. Insoluble material in the assay mixture was removed by centrifugation at 5,000 rpm, $30 \mathrm{~min}$ and the absorbance was read at $495 \mathrm{~nm}$ using a double beam uv-vis spectrophotometer (UV5704SS, India).

Inhibition of elastase activity by fungal agents (oils at 1:10 dilution) was evaluated using the modified method of Okumura et al. (2007). The known inhibitors of proteinases namely, ethylene diamine tetra acetic acid (EDTA), $5 \mathrm{mM}$; and metal ion $\mathrm{Mg}^{2+}\left(\mathrm{MgSO}_{4}\right), 1 \mathrm{mM}$; were also used in the study. In the assay, $200 \mu \mathrm{L}$ of test sample was mixed with $50 \mu \mathrm{L}$ of inhibitor solution and incubated at $37{ }^{\circ} \mathrm{C}$ for $45 \mathrm{~min}$ before adding to elastin congo red substrate mixture. Further, procedure was followed as performed for elastase activity. Test sample without exposure to inhibitor was run as control. All the experiments were done three times in triplicate and the mean test absorbance value was subtracted from the mean untreated control absorbance value to obtain the percent reduction in elastase activity.

\section{Detection and inhibition of keratinase activity}

Estimation of keratinase activity in tested fungi was performed using the modified method of Muhsin and Aubaid (2000). Briefly, $500 \mu \mathrm{L}$ of cell free supernatant was mixed with $50 \mathrm{mg}$ of guinea pig hair in $5 \mathrm{~mL}$ of $0.03 \mathrm{M}$ phosphate buffer (pH 7.8) and incubated at $37^{\circ} \mathrm{C}$ for $3 \mathrm{~h}$, $150 \mathrm{rpm}$. Test sample was replaced by buffer to run the control. Reaction mixture was stopped by adding $5 \mathrm{~mL}$ of $10 \%$ TCA and kept on ice for $30 \mathrm{~min}$ and centrifuged at $5,000 \mathrm{rpm}$ for $30 \mathrm{~min}$. The hair was removed by filtration and the absorbance was read at $280 \mathrm{~nm}$ using a double beam uv-vis spectrophotometer (UVS504SS, India).
Inhibitory effect of test agents was evaluated by the method of Okumura et al. (2007) with some modifications, and employing known inhibitors of proteinases as used in elastase inhibition assays. In the assay, $400 \mu \mathrm{L}$ of test sample was mixed with $100 \mu \mathrm{L}$ of inhibitor solution and incubated at $37{ }^{\circ} \mathrm{C}$ for $45 \mathrm{~min}$. The control was run without inhibitors. Each experiment was performed three times in triplicate and inhibition of keratinase activity was calculated in terms of percent reduction in mean absorbance value of test sample compared with untreated control.

\section{Statistical analysis}

All the experiments were performed three times with three replicates per experiment and data are expressed as mean \pm standard deviation. Statistical significance of the differences was determined by the one way ANOVA test using Minitab (V.11.0 for Windows). \% reduction in elastase and keratinase activity in test strains in the presence of oils/inhibitors was compared to untreated control by one way ANOVA using Duncan's method. P-values of $\leq 0.05$ were considered as statist \pm ally significant.

\section{Results}

\section{GC-GC/MS analysis}

GC and GC-MS analysis of essential oils revealed the presence of various major and minor compounds. Main $C$. copticum oil components were $\rho$-cymene (33.67\%), thymol $(22.82 \%)$ and $\gamma$-terpinene $(21.61 \%)$. Thymol was the main constituent (44.71\%) of $T$. vulgaris followed by $\gamma$-terpinene (26.01\%) and $\alpha$-cymene $(21.22 \%)$.

\section{Antifungal activity}

MIC of fluconazole was found to be $200 \mu \mathrm{g} / \mathrm{mL}$ against $A$. fumigatus MTCC2550 and A. niger IOA3 whereas $800 \mu \mathrm{g} / \mathrm{mL}$ against $T$. rubrum IOA9. Both the tested oils exhibited MIC of $72 \mu \mathrm{g} / \mathrm{mL}$ and $144 \mu \mathrm{g} / \mathrm{mL}$ against T. rubrum IOA9 and A. fumigatus MTCC2550, respectively. MICs of $T$. vulgaris and C. copticum were $288 \mu \mathrm{g} / \mathrm{mL}$ and $576 \mu \mathrm{g} / \mathrm{mL}$ against $A$. niger IOA3, respectively. Thymol exhibited MIC of $192 \mu \mathrm{g} / \mathrm{mL}$ against the strains $A$. fumigatus MTCC2550 and T. rubrum IOA3 whereas $384 \mu \mathrm{g} / \mathrm{mL}$ against $A$. niger IOA3.

Furthermore, tested oils exerted concentration dependent inhibitory effects on the production of fungal biomass (Table 1) and mycelial radial growth (Table 2). Thymol was the most inhibitory resulting in reduction in the biomass as well as radial growth from $94.07 \%$ to $99.75 \%$ in tested strains at $72 \mu \mathrm{g} / \mathrm{mL}$. Reference drug fluconazole also exhibited concentration dependent inhibition in biomass and radial growth of tested fungi up to $84.89 \%$ and $87.08 \%$, respectively at $200 \mu \mathrm{g} / \mathrm{mL}$. 

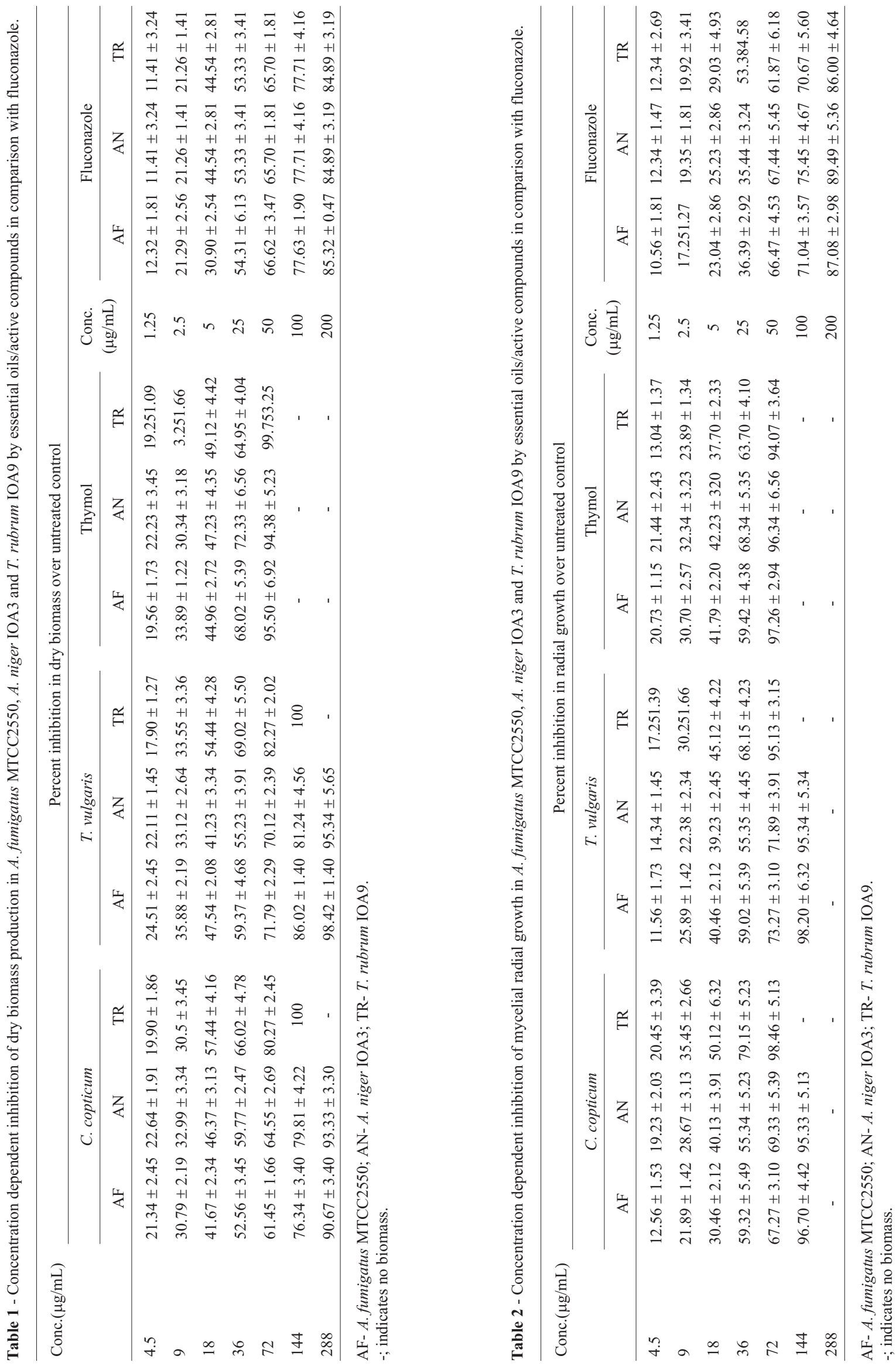


\section{Cellular toxicity assay}

Tested agents showed no haemolysis in vitro at their respective MFCs. Only at a concentration two to four times higher of MFCs $(2304 \mu \mathrm{g} / \mathrm{mL})$ partial haemolysis (10$25 \%$ ) was observed. Complete haemolysis was shown at $0.1 \%$ (v/v) Triton X-100 (positive control) and no haemolysis was observed by $1 \%$ DMSO and PBS (solvent controls) (Table 3).

\section{Synergy of essential oils with fluconazole}

T. vulgaris and thymol showed significant levels of synergistic interaction with fluconazole against $T$. rubrum IOA9 and A. fumigatus MTCC2550, whereas, C. copticum interacted indifferently with fluconazole against the tested fungi (Table 4). Thymol exhibited the highest synergy with FICI values of 0.187 and 0.156 against A. fumigatus MTCC2550 and T. rubrum IOA9, respectively.

\section{Susceptibility of macroconidia and arthroconidia}

As shown in Table 5, the MICs of eugenol and $T$. vulgaris were found to be $72 \mu \mathrm{g} / \mathrm{mL}$ against both macro and arthroconidia and MICs of C. copticum was only 2-fold higher against arthroconidia, in comparison with macro-
Table 5 - Effect of essential oils and active compounds in terms of MIC against arthroconidia compared to macroconidia in T. rubrum IOA9.

\begin{tabular}{lcc}
\hline \multirow{2}{*}{ Test agents } & \multicolumn{2}{c}{ MIC $(\mu \mathrm{g} / \mathrm{mL})$} \\
\cline { 2 - 3 } & Macroconidia & Arthroconidia \\
\hline Essential oils & 72 & 144 \\
Carum copticum & 72 & 72 \\
Thymus vulgaris & & 72 \\
Active compounds & 72 & \\
Thymol & & 1600 \\
Antifungal drugs & 200 & \\
Fluconazole & & \\
\hline
\end{tabular}

conidia. Control drug fluconazole was not much effective, as MIC against arthroconidia was 8-fold higher compared with macroconidia.

\section{Inhibition of elastase and keratinase activity}

Since $A$. niger IOA3 and T. rubrum IOA9 showed relatively higher production of elastase and keratinase, respectively, the essential oils were only tested for their anti-elastase and anti-keratinase activity against these two strains. As shown in Table 6, thymol showed the highest re-

Table 3 - Haemolytic properties of C. copticum, T. vulgaris oils and thymol.

\begin{tabular}{|c|c|c|c|c|c|c|c|}
\hline \multirow[t]{3}{*}{ Test agents } & \multicolumn{7}{|c|}{ Percent haemolysis of RBC (Mean \pm SD) } \\
\hline & \multicolumn{7}{|c|}{ Concentration of essential oils/active compounds ( $\mu \mathrm{g} / \mathrm{mL}$ ) } \\
\hline & 18 & 36 & 72 & 144 & 288 & 576 & 2304 \\
\hline \multicolumn{8}{|l|}{ Essential oils } \\
\hline C. copticum & $1.48 \pm 0.14$ & $1.84 \pm 0.14$ & $2.44 \pm 0.19$ & $3.51 \pm 0.07$ & $4.27 \pm 0.17$ & $4.62 \pm 0.27$ & $21.03 \pm 0.30$ \\
\hline T. vulgaris & $1.38 \pm 0.14$ & $1.84 \pm 0.14$ & $2.74 \pm 0.19$ & $3.21 \pm 0.07$ & $4.09 \pm 0.17$ & $4.92 \pm 0.27$ & $19.11 \pm 0.40$ \\
\hline \multicolumn{8}{|c|}{ Active compounds } \\
\hline Thymol & $1.64 \pm 0.19$ & $2.59 \pm 0.07$ & $3.45 \pm 0.12$ & $3.99 \pm 0.05$ & $4.68 \pm 0.17$ & $5.66 \pm 0.20$ & $16.34 \pm 0.23$ \\
\hline
\end{tabular}

Table 4 - FIC and FICI for combination of essential oils or active compounds with fluconazole against A. fumigatus MTCC2550 and T. rubrum IOA9.

\begin{tabular}{|c|c|c|c|c|c|c|c|c|}
\hline \multirow[t]{2}{*}{ Test combinations } & \multicolumn{4}{|c|}{ A. fumigatus MTCC2550 } & \multicolumn{4}{|c|}{ T. rubrum IOA9 } \\
\hline & $\mathrm{MIC}_{\mathrm{a}}$ & $\mathrm{MIC}_{\mathrm{c}}$ & FICI & $\mathrm{T}$ & $\mathrm{MIC}_{\mathrm{a}}$ & $\mathrm{MIC}_{\mathrm{c}}$ & FICI & $\mathrm{T}$ \\
\hline \multicolumn{9}{|c|}{ C. copticum with fluconazole } \\
\hline C. copticum $(\mu \mathrm{g} / \mathrm{mL})$ & 144 & 9 & 0.562 & I & 72 & 18 & 0.75 & I \\
\hline Fluconazole $(\mu \mathrm{g} / \mathrm{mL})$ & 200 & 100 & & & 200 & 100 & & \\
\hline \multicolumn{9}{|c|}{ T. vulgaris with fluconazole } \\
\hline T. vulgaris $(\mu \mathrm{g} / \mathrm{mL})$ & 144 & 18 & 0.250 & $\mathrm{~S}$ & 72 & 9 & 0.250 & $\mathrm{~S}$ \\
\hline Fluconazole $(\mu \mathrm{g} / \mathrm{mL})$ & 200 & 25 & & & 200 & 25 & & \\
\hline \multicolumn{9}{|c|}{ Thymol with fluconazole } \\
\hline Thymol ( $\mu \mathrm{g} / \mathrm{mL})$ & 192 & 12 & 0.187 & $\mathrm{~S}$ & 192 & 6 & 0.156 & $\mathrm{~S}$ \\
\hline Fluconazole $(\mu \mathrm{g} / \mathrm{mL})$ & 200 & 25 & & & 200 & 25 & & \\
\hline
\end{tabular}

$\mathrm{MIC}_{\mathrm{a}}$, MIC of one agent alone; $\mathrm{MIC}_{\mathrm{c}}$, MIC of agent in most effective combination; I- indifferent; S- synergy. 
Table 6 - Effect of essential oils and active compounds on elastase and keratinase activities in A. niger IOA3 and T. rubrum IOA9, respectively.

\begin{tabular}{lcc}
\hline Test agents & $\begin{array}{l}\text { Percent reduction in } \\
\text { absorbance at } 495 \mathrm{~nm} \\
\text { over untreated control } \\
\text { for elastase activity }\end{array}$ & $\begin{array}{l}\text { Percent reduction in } \\
\text { absorbance at } 280 \mathrm{~nm} \\
\text { over untreated control } \\
\text { for keratinase activity }\end{array}$ \\
\hline $\begin{array}{l}\text { Essential oils } \\
\text { C. copticum }\end{array}$ & $81.24 \pm 3.89$ & $24.33 \pm 1.24$ \\
T. vulgaris & $90.75 \pm 3.45$ & $15.96 \pm 1.23$ \\
Active compounds & $95.56 \pm 4.09$ & $13.90 \pm 1.22$ \\
Thymol & & \\
Inhibitors & $72.63 \pm 1.26$ & $54.48 \pm 2.20$ \\
EDTA & $94.10 \pm 1.63$ & $39.77 \pm 1.06$ \\
$\mathrm{MgSO}_{4}$ & & \\
\hline
\end{tabular}

duction (95.56\%) in comparison with untreated controls in elastase activity followed by $T$. vulgaris $(90.75 \%)$ and $C$. copticum (81.24\%). On the contrary, tested oils were less effective in reducing the keratinase activity.

\section{Discussion}

The present in vitro study explored efficacy of oils of C. copticum and T. vulgaris and their major active constituent thymol in inhibiting growth and virulence against the azole-resistant strains of Aspergillus spp. and T. rubrum. Tested oils/compound exerted concentration dependent inhibitory effects on the production of fungal biomass and mycelial radial growth. Thymol was the major active ingredient of both the oils as revealed by GC/GC-MS analysis. This indicates that inhibitory activity of the oils of $C$. copticum and T. vulgaris may be due to the thymol. However, minor constituents may also play a key role in the biological activities of these oils. Further, oils of $C$. copticum, T. vulgaris and thymol were tested for their toxicities to sheep erythrocytes. An alternative approach to combat drug-resistant strains is the use of antifungal agents in combination to reduce host toxicity and emergence of resistance. The promising in vitro antifungal activity of tested agents along with their non-toxicity to RBCs, prompted us to investigate in vitro combinations of these essential oils and thymol with fluconazole to increase efficacy of this drug against the azole-resistant strains. To our knowledge, no investigation for these combinations has been carried out before.

T. vulgaris and thymol showed significant levels of synergistic interaction with fluconazole against tested fungi, whereas, C. copticum interacted indifferently. Thymol exhibited highest synergy with fluconazole against A. fumigatus MTCC2550 (FICI values of 0.187) and $T$. rubrum IOA9 (0.156), respectively. Level of synergy of $T$. vulgaris with fluconazole was comparatively lesser, with the FICI value of 0.250 . This is probably due to the pres- ence of $\alpha$-cymene (21.22\%) and $\gamma$-terpinene (26.01\%) that has diluted the synergistic effect of thymol (44.71\%) compared to pure compound. The amount of thymol in $C$. copticum is $22.82 \%$ that is almost two fold lesser compared to amount present in T. vulgaris and it could have probably further reduced the synergistic effect of thymol and resulted in indifference type of interaction with fluconazole. Thymol reduced the MIC of fluconazole up to 4-fold and its MIC decreased up to 16- and 32-folds against $A$. fumigatus MTCC2550 and T. rubrum IOA9, respectively. Treatment of fungal infections caused by the drug-resistant fungi may require higher doses in monotherapy and if fluconazole is used in higher concentration it can lead to adverse side effects such as hepatotoxicity (Groll et al., 1998). In these perspectives, these data suggests that thymol could be quite effectively used in combating dose-related toxicity and drug-resistance against fluconazole in the treatment of these fungi.

Infections caused by filamentous fungi are characterized by the presence of hyphal elements in host tissues, however, formation of arthroconidia constitute the primary mode of transmitting dermatophytic infections such as onychomycosis in humans and animals (Malten and Thiele, 1973; Gupta et al., 2003). Arthroconidia are more resistant to antifungals and may be one of the causes of therapeutic failure, mainly in patients whose lesions contain abundant arthroconidia (Miyazi and Nishimura, 1971). Generally, in vitro testing evaluates the responses of microconidia, macroconidia or hyphae (Arrese et al., 2001; Yazdanparast and Barton, 2006) but under in vivo conditions infectious entity is mostly found to be arthroconidia (Yazdanparast and Barton, 2006). Hence, there is a need of susceptibility testing for antifungal agents against arthroconidia in order to determine their effectiveness at infection sites. Therefore, we compared MIC of tested oils and fluconazole against macroconidia and arthroconidia in $T$. rubrum IOA9. Tested oils/compounds showed inhibitory activity against arthroconidia at par compared with fluconazole. Arthroconidia and macroconidia of T. rubrum IOA9 were equally susceptible to T. vulgaris and thymol. However, arthroconidia was found to be more tolerant to fluconazole in comparison with macroconidia. These data account for the effectiveness of thymol and oil of $T$. vulgaris containing higher amount of thymol (44.71\%) in treating dermatophytic infections. In our knowledge, efficacy of these oils and compounds against arthroconidia has not been reported before.

The systematic development of active pharmaceutical ingredients addressing virulence factors as a prime target could provide entirely novel therapeutic options for the treatment and/or prevention of localized or systemic fungal disease. Targeting virulence factors is now gaining interest as an alternative strategy to develop new anti-infective agents. The increasing trend of screening of plant products for their anti-virulent activity against fungi prompted us to 
evaluate some of these oils for their anti-pathogenic efficacy. Production of elastases and keratinases are reported to aid in the pathogenesis of Aspergillus spp. and Trichophyton spp. (Okumura et al., 2007, Vermout et al., 2008) and, therefore inhibition of elastase and keratinase activities in A. fumigatus and T. rubrum was determined by these oils. Tested oils showed $>80 \%$ reduction in the elastase activity of $A$. fumigates MTCC2550. However, these oils appeared to be less effective in reducing the keratinase activity. Since proteinases contribute to fungal virulence by destroying host tissues and digesting immunologically important proteins such as antibodies and complement factors (Santos et al., 2007), the inhibition of these enzyme activities in particular elastase and anti-arthroconidial activity may effectively reduce the pathogenesis of A. fumigatus.

\section{Conclusions}

In our study, the novel anti-proteinase activity of the tested oils in addition to mycelial growth inhibition, and effectiveness against drug tolerant arthroconidia are indicative of their efficacy as potential antifungal drugs. The data obtained have highlighted the T. vulgaris as more inhibitory compared with C. copticum and this activity appeared to be correlated to the presence of high amount of thymol in the $T$. vulgaris oil. Moreover thymol and T. vulgaris containing higher amount of thymol also exhibited strong synergistic interactions with fluconazole against the tested fungi. This could highlight the potential exploitation of thymol in combination therapy for treatment of drugresistant strains of $A$. fumigatus and $T$. rubrum. The current studies draw attention for developing new compounds especially thymol as antifungal agents in the management of aspergillosis and dermatophytosis. However, in vivo evaluations are needed to unfold the therapeutic potential of these agents in combating such fungal diseases.

\section{Acknowledgement}

We are grateful to Advanced Instrumentation and Research Facility at Jawaharlal Nehru University, New Delhi for helping in GC/GC-MS analysis. We are also thankful to Indian Council of Medical Research, New Delhi, for financial assistance in the form of senior research fellowship to MSAK.

\section{Conflict of interest}

Authors declare no conflict of interest.

\section{References}

Ahmad I, Khan MSA, Zahin M, Owais M, Shahid M, Mehmood Z, Pant AB (2010) Combinational antifungal therapy and recent trends in drug discovery. In: Ahmad, I.; Owais, M.; Shahid, M.; Aqil, F. (eds). Combating Fungal Infections:
Problems and Remedy, Springer-Verlag, Berlin Heidelberg, Germany, pp 213-240.

Arrese JE, Pierard-Franchimont C, Pierard GE (2001) A plea to bridge the gap between antifungals and the management of onychomycosis. Am J Clin Dermatol 2:281-284.

Baddley JW, Pappas PG (2007) Combination antifungal therapy for the treatment of invasive yeast and mold infections. Curr Infect Dis Rep 9:448-456.

Bairwa R, Sodha RS, Rajawat BS (2012) Trachyspermum ammi. Pharmacogn Rev 6:56-60.

Bajpai VK, Yoon JI, Kang SC (2009) Antifungal potential of essential oil and various organic extracts of Nandina domestica Thunb against skin infectious fungal pathogens. Appl Microbiol Biotechnol 83:1127-1133.

Barker KS, Rogers PD (2006) Recent insights into the mechanisms of antifungal resistance. Curr Infect Dis Rep 8:28162823.

Barros MES, Santos DA, Hamdan JS (2007) Evaluation of susceptibility of Trichophyton mentagrophytes and Trichophyton rubrum clinical isolates to antifungal drugs using a modified CLSI microdilution method (M38-A). J Med Microbiol 56:514-518.

Cavaleiro C, Pinto E, Goncalves MJ, Salgueiro L (2006) Antifungal activity of Juniperus essential oils against dermatophyte, Aspergillus and Candida strains. J Appl Microbiol 100:1333-1338.

Dagenais TRT, Keller NP (2009) Pathogenesis of Aspergillus fumigatus in invasive aspergillosis. Clin Microbiol Rev 22:447-465.

Davies NW (1990) Gas chromatographic retention indices of monoterpenes and sesquiterpenes on methyl silicone and carbowax $20 \mathrm{M}$ phases. J Chromatogr A 503:1-24.

Gauwerky K, Borelli C, Korting HC (2009) Targeting virulence: a new paradigm for antifungals. Drug Discov Today 14:214222.

Groll AH, Piscitelle SC, Walsh TJ (1998) Clinical pharmacology of systemic antifungal agents: a comprehensive review of agents in clinical use, current investigational compounds, and putative targets for antifungal drug development. Adv Pharmacol 44:343-500.

Gupta AK, Ahmad I, Porretta M, Summerbell RC (2003) Arthroconidial formation in Trichophyton raubitschekii. Mycoses 46:304-310.

Khan MSA, Ahmad I (2011) Antifungal activity of essential oils and their synergy with fluconazole against drug resistant strains of Aspergillus fumigatus and Trichophyton rubrum. Appl Microbiol Biotechnol 90:1083-1094.

Kothary MH, Chase TJr, Macmillan JD (1984) Correlation of elastase production by some strains of Aspergillus fumigatus with ability to cause pulmonary invasive aspergillosis in mice. Infect Immun 43:320-325.

Luize PS, Tiuman TS, Morello LG, Maza PK, Ueda-Nakamura T, Filho BPD, Cortez DAG, de Mello JCP, Nakamura CV (2005) Effects of medicinal plant extracts on growth of Leishmania (L.) amazonensis and Trypanosoma cruzi. Braz J Pharm Sci 41:1-10.

Malten KE, Thiele FA (1973) Evaluation of skin damage. II. Water loss and carbon-dioxide release measurements related to skin resistance measurements. Braz J Dermatol 89:565-569.

Miyazi M, Nishimura K (1971) Studies on arthrospores of Trichophyton rubrum. II. Relationship between the types of 
eruption and the parasitic forms of Trichophyton rubrum. Jpn J Med Mycol 12:81-85.

Monod M (2008) Secreted proteases from dermatophytes. Mycopathologia 166:285-294.

Muhsin TM, Aubaid AH (2000) Partial purification and some biochemical characteristics of exocellular keratinase from Trichophyton mentagrophytes var. erinacei. Mycopathologia 150:121-125.

Odds FC (2003) Synergy, antagonism, and what the chequerboard puts between them. J Antimicrob Chemother 52:1.

Okumura Y, Ogawa K, Uchgiya K, Nikai T (2007) Characterization and primary structure of elastase inhibitor, AFLEI from Aspergillus flavus. Jpn J Med Mycol 18:13-18.

Pina-Vaz C, Rodrigues AG, Pinto E, Costa-de-Oliveira S, Tavares C, Salgueiro L, Cavaleiro C, Goncalves MJ, Martinezde-Oliveira J (2004) Antifungal activity of Thymus oils and their major compounds. Eur Acad Dermatol Venereol 18:73-78.

Quiroga EN, Sampietro AR, Vattuone MA (2004) In vitro fungitoxic activity of Larrea divaricata cav. extracts. Lett Appl Microbiol 39:7-12.

Sachar LA, Winter KK, Sicher N, Frankel S (1955) Photometric method for estimation of elastase activity. Proc Soc Exp Biol Med 90:323-326.

Santos ALS, Palmeira VF, Rozental S, Kneipp LF, Nimrichter L, Alviano DS, Rodrigues ML, Alviano CS (2007) Biology and pathogenesis of Fonsecaeaa pedrosoi, the major etiologic agent of Chromoblastomycosis. FEMS Microbiol Rev 31:570-591.

Shafique S, Shafique S, Bajwa R, Akhtar N, Hanif S (2011) Fungitoxic activity of aqueous and organic solvent extracts of Tagetes erectus on phytopathogenic fungus- Ascochyta rabiei. Pak J Bot 43:59-64.
Sokovic M, Glamoclija J, Ciric A, Kataranovski D, Marin PD, Vukojevic J, Brkic D (2008) Antifungal activity of the essential oil of Thymus vulgaris L. and thymol on experimentally induced dermatomycoses. Drug Dev Ind Pharm 34:1388-1393.

Tullio V, Nostro A, Mandras N, Dugo P, Banche G, Cannatelli MA, Cuffini AM, Alonzo V, Carlone NA (2007) Antifungal activity of essential oils against filamentous fungi determined by broth microdilution and vapour contact methods. J Appl Microbiol 102:1544-1550.

Vazquez JA (2003) Combination antifungal therapy against Candida species: the new frontier - are we there yet? Med Mycol 41:355-368.

Venkatesan G, Singh AJAR, Murugesan AG, Janaki C, Shankar SG (2007) Trichophyton rubrum- the predominant etiological agent in human dermatophytoses in Chennai, India. Afr J Microbiol Res 1:9-12.

Vermout S, Tabart J, Baldo A, Mathy A, Losson B, Nignon B (2008) Pathogenesis of dermatophytosis. Mycopathologia 166:267-275.

Voltan AR, Donofrio F, Miranda ET, Moraes RA, Mendes-Giannini MJS (2008) Induction and secretion of elastinolytic and proteolytic activity in cultures of Paracoccidioides brasiliensis. J Basic Appl Pharm Scie 29:97-106.

Yazdanparast SA, Barton RC (2006) Arthroconidia production in Trichophyton rubrum and a new ex vivo model of onychomycosis. J Med Microbiol 55:1577-1581.

Zacchino S, Lopez S, Pezzenati G, Furlan R, Santecchia C, Munoz L, Gianni F, Rodriguez A, Enriz R (1999) In vitro evaluation of antifungal properties of phenylpropanoids and related compounds acting against dermatophytes. J Nat Prod 63:1353-1357.

All the content of the journal, except where otherwise noted, is licensed under a Creative Commons License CC BY-NC. 